\title{
La ciudad enferma: Espacio, metáfora y mito en Manhattan Transfer, de John Dos Passos
}

\author{
Rebeca Gualberto VALVERDE \\ Departamento de Filología Inglesa II \\ Universidad Complutense de Madrid \\ rebeca.gualberto@gmail.com
}

Recibido: 6 de noviembre de 2010

Aceptado: 21 de febrero de 2011

\begin{abstract}
Resumen
Por cuanto la experiencia urbana no puede desligarse de la cultura occidental contemporánea, el estudio de las representaciones literarias de la ciudad resulta crucial para analizar el Zeitgeist cultural e ideológico de una determinada época. De manera específica, este artículo tiene como objetivo explorar la retórica de la enfermedad en la representación urbana que lleva a cabo Manhattan Transfer (1925), de John Dos Passos, desde una perspectiva simbólica y mito-crítica. Debe para ello tenerse en cuenta la existencia de un fuerte sentimiento de hostilidad hacia el espacio urbano en la literatura modernista estadounidense, sobre todo en textos ambientados en la ciudad de Nueva York -"that most mythical of cities, [which] tends to emerge in recent literature as hellish, or at any rate murderous" (Oates 1981: 30). En la novela de Dos Passos, Nueva York aparece representada como el espacio enfermo de la modernidad. Tal representación da cuenta de una relación de continuidad entre el espacio y la comunidad que lo habita, pues no debe ignorarse que el espacio urbano funciona como sistema de organización de un conjunto de relaciones sociales entre individuos y, en consecuencia, como correlato simbólico de todas aquellas instituciones comunitarias que articulan las relaciones entre el individuo y el conjunto de la sociedad. Así, un espacio (mítico) enfermo -la megalópolis moderna- se configura en el texto modernista como la extensión metafórica de una comunidad enferma.
\end{abstract}

Palabras clave: John Dos Passos, Manhattan Transfer, enfermedad, mitocrítica, modernismo de los Estados Unidos.

Title: The sick city: Space, Metaphor, and Myth in John Dos Passos' Manhattan Transfer

\section{Abstract}

Insofar as urban experience is inextricably related to contemporary Western culture, the study of literary urban representations becomes crucial in order to analyze the cultural and ideological zeitgeist of a particular age. Specifically, this article aims to explore the rhetoric of sickness in John Dos Passos' Manhattan Transfer (1925), from a symbolic and myth-critical perspective. It this regard, it should be born in mind the fact that American modernist literature is characterized by a generalized hostility towards urban spaces. Such hostility is especially obvious in texts focusing on New York City -"that most mythical of cities, [which] tends to emerge in recent literature as hellish, or at any rate murderous" (Oates 1981: 30). In 
Manhattan Transfer, New York is indeed represented as murderous, inasmuch as it is represented as the sick space of modernity. Such rendering of the city accounts for a relationship of symbolic continuity between urban spaces and urban communities. As this article examines, the modern city functions as an organizational system of human relations and, therefore, as the emblematic correlative of communitarian institutions. Arguably, Manhattan Transfer thus shapes a (mythic) sick space as the metaphorical extension of a sick urban community.

Keywords: John Dos Passos, Manhattan Transfer, sickness, myth-criticism, American Modernism.

En The city in Literature, Richard Lehan escribe: "The city has determined our cultural fate for the last three hundred years-has become inseparable from our personal and national destiny. As the product of the Enlightenment, urbanism is at the very heart of Western culture, the source both of political order and of social chaos" (Lehan 1998: 3). Así pues, en tanto que la experiencia urbana no puede desligarse de la cultura occidental contemporánea, el estudio de las representaciones literarias de la ciudad resulta crucial para analizar el Zeitgeist cultural e ideológico de una determinada época. De manera específica, este artículo tiene como objetivo explorar la retórica de la enfermedad en la representación urbana que lleva a cabo Manhattan Transfer (1925), de John Dos Passos, desde una perspectiva simbólica y mitocrítica. En efecto, dicha retórica de la enfermedad confirma que, como apunta Lehan, "as the city became more materialistic, it engendered a hostility in the literary imagination" (Lehan 1998: 5) ${ }^{1}$. Tal hostilidad se manifiesta de manera muy elocuente en la literatura modernista estadounidense, sobre todo en textos ambientados en la ciudad de Nueva York ${ }^{2}$. Tal y

\footnotetext{
${ }^{1}$ Leslie Fielder coincide: "Somehow we grow impatient with cities themselves [...]. But the disease, the impatient of which I speak, existed in the literature bred by our deepest nightmares long before the Industrial Revolution had radically transformed the more humane poli into an impersonal hub of communications, a center for mass production, marshalling yards, slaughter houses, and assembly plants. At first, indeed, that transformation seemed a blessing rather than a curse for the city poor, since it created more work, more goods, and eventually lifted more men and women above the subsistence level. But simultaneously it raised expectations even higher, making those still excluded and deprived ever more aware of their suffering, while rich and poor alike became conscious of the price paid: the growing alienation of all humankind from the natural world in which we first become human, and which in turn we have humanized by making it a part of our essential mythology, the perceptual grid through which we see and understand our identity and destiny" (Fielder 1981: 114; mi cursiva).

2 Junto con Manhattan Transfer, otro texto fundamental para analizar cómo la retórica de la enfermedad y de la degeneración se vincula a la ciudad de Nueva York es The Great Gatsby (1925), de Francis Scott Fitzgerald. No obstante, la prominencia de Nueva York sobre otras ciudades en la literatura estadounidense no es exclusiva del modernismo. Por el contrario, la ciudad de Nueva York se configura
} 
como afirma Joyce Carol Oates: "New York City-that most mythical of cities-tends to emerge in recent literature as hellish, or at any rate murderous" (Oates 1981: 30; mi cursiva). En efecto, ocurre así en Manhattan Transfer.

Según puede argumentarse, en la novela de Dos Passos Nueva York aparece representada como el espacio enfermo de la modernidad. A este respecto, debe notarse primero la metáfora inherente a dicha representación. Si la ciudad puede enfermar, entonces debe identificarse con un cuerpo o, al menos, con un organismo dotado de vida. En segundo lugar, por cuanto el espacio urbano en la literatura constituye una suerte de heterotopía foucaultiana $^{3}$-la ciudad en la literatura es una representación simbólica (irreal) de un espacio real (Scott y Simpson-Housley 1994: 335)- debe tenerse en consideración que, en consecuencia, la recreación literaria del espacio urbano debe configurarse en función de una perspectiva ideológica determinada. Así, toda representación literaria de la ciudad ofrece, según puede argüirse, una crítica de las condiciones sociales, morales y políticas de un espacio urbano real (Simpson-Housley y Scott 1994: 335). No debe entonces ignorarse que el espacio urbano funciona como sistema de organización de un conjunto de relaciones sociales entre individuos $y$, en consecuencia, como correlato simbólico de todas aquellas instituciones comunitarias que articulan las relaciones entre el individuo y el conjunto de la sociedad ${ }^{4}$. Así, un espacio enfermo -se hace preciso en este punto trascender los límites de la metáfora que atribuye una cualidad orgánica a un espacio, por condición, inerte- se configura como la extensión semántica de una comunidad enferma. Precisamente, la retórica de la enfermedad en Manhattan Transfer sirve para establecer tal relación de continuidad entre el espacio y la comunidad que lo habita.

No obstante, antes de analizar cuál es la enfermedad que padece la comunidad urbana de Manhattan Transfer, y cómo dicha

como marco y foco de muchos de los textos fundamentales de finales del siglo XIX y principios del XX. Tal es el caso de, entre otros, Washington Square (1880), de Henry James; Maggie: a Girl of the Streets (1983), de Stephen Crane; The House of Mirth (1905), de Edith Wharton, o An American Tragedy (1925), de Theodore Dreiser.

3 "Hay de igual modo, y probablemente en toda cultura, en toda civilización, espacios reales, espacios efectivos, espacios delineados por la sociedad misma, y que son una especie de contraespacios, una especie de utopías efectivamente verificadas en las que los espacios reales, todos los demás espacios reales que pueden hallarse en el seno de una cultura están a un tiempo representados, impugnados o invertidos, una suerte de espacios que están fuera de todos los espacios, aunque no obstante sea posible su localización" (Foucault 1997: par. 13).

4 "[...] vivimos en el interior de un conjunto de relaciones que determinan ubicaciones mutuamente irreductibles y en modo alguno superponibles" (Foucault 1997: par. 10). 
enfermedad contagia al espacio de la ciudad, se hace preciso vincular la novela de Dos Passos a otros textos contemporáneos. Al respecto de esto, debe notarse que Manhattan Transfer es una manifestación muy elocuente del espíritu de su tiempo, de manera particular por lo que respecta a la relación entre literatura y espacio urbano. Como ya se ha mencionado, el Zeitgeist modernista resulta especialmente vehemente en la representación de un sentimiento moderno de hostilidad $-y$, no debe olvidarse, fascinación (si bien, en no pocas ocasiones, morbosa)- hacia la ciudad. Así, la novela de Dos Passos da cuenta de una percepción de la ciudad de Nueva York como un espacio de enfermedad que está también presente en otros textos influyentes del momento. Éste es el caso de la autobiografía del historiador y filósofo estadounidense Henry Adams (1838-1918). En The Educaction of Henry Adams (1918), el autor describe Nueva York como sigue:

The outline of the city became frantic in its effort to explain something that defied meaning. Power seemed to have outgrown its servitude and to have asserted its freedom. The cylinder had exploded, and thrown great masses of stone and steam against the sky. The city had the air and movement of hysteria, and the citizens were crying, in every accent of anger and alarm, that the new forces must at any cost be brought under control. Prosperity never before imagined, power never yet wielded by man, speed never reached by anything but a meteor, had the world irritable, nervous, querulous, unreasonable and afraid. (Adams 1961: 499; mi cursiva)

Henry Adams relata su llegada a Nueva York el 5 de noviembre de 1904, esto es, unos años después de las primeras escenas de Manhattan Transfer, y más de dos décadas antes de la publicación de la novela, en 1925. El desarrollo cronológico de Manhattan Transfer abarca desde finales del siglo XIX hasta la década de 1920, y reproduce, desde una perspectiva simbólica, el recrudecimiento de todos aquellos elementos deshumanizantes e histéricos que menciona Adams en su descripción de la ciudad. Cabe establecer por tanto una conexión entre modernidad, espacio urbano y enfermedad que caracteriza el Zeitgeist modernista. Desde esta perspectiva, la mención de la histeria por parte de Adams resulta especialmente elocuente. En 1904, tal y como describe Adams, Nueva York se percibía y se representaba ya como una ciudad enferma. No es descabellado especular que, quizá, dos décadas más tarde, cuando John Dos Passos publica Manhattan Transfer, la enfermedad histérica de la ciudad podría haberse agravado. Algunos testimonios historiográficos refuerzan esta idea. Por ejemplo, el historiador William E. Leuchtenburg describe así cómo era Nueva York en la década de los años veinte: 
The city, rural traditionalists expounded, was the home of the alien and the uprooted Negro, of a people lost to fundamental American values. 'New York', wrote the Denver Post in 1930, 'has been a cesspool into which immigrant trash has been dumped for so long that it can scarcely be considered American anymore.' New York was the seat of the Union Theological Seminary and modernism, the home of the nightclub and the gangster, of Wall Street and Tammany Hall [...]. It was a city cruel and impersonal, the abode of the rootless, a place where, as one writer noted, 'nobody seemed to have parents' ${ }^{5}$. (Leuchtenburg 1958: 226-227)

Nueva York, histérica en 1904, se ha convertido en la década de 1920 en "la morada de los desarraigados". Constituye por tanto no sólo un espacio enfermo, sino también un espacio de desarraigo, en el que nada ni nadie puede crecer o echar raíces. Se trata, desde esta perspectiva, de una tierra yerma, real y simbólica a un tiempo. En "The Cities of Modernism," Malcolm Bradbury explica que, por lo que al modernismo se refiere, "the city has become metaphor rather than place" (Bradbury 1991: 97). La ciudad moderna, histérica y yerma, funciona a la vez como espacio y como metáfora. Es más, funciona más como metáfora que como espacio. Cabe preguntarse, sin embargo, cuál es el término real de dicha metáfora. Como ya se ha apuntado, este estudio argumenta que el espacio urbano del texto modernista es la metáfora que define y configura a la comunidad que lo habita -esto es, una comunidad uniformemente afectada por una enfermedad colectiva.

La comunidad urbana de la modernidad, siguiendo la explicación de Leuchtenburg, es una comunidad marcada, primero, por el desarraigo. De manera muy elocuente, tal condición existencial de desarraigo se representa -no ya metafóricamente, sino desde una perspectiva mítica- mediante la reinterpretación del mito premoderno de la Tierra Baldía, esto es, el mito por excelencia del modernismo de los Estados Unidos ${ }^{6}$. Al respecto de esto, recuérdese

\footnotetext{
5 "It [America] wasn't our country any longer. Nevertheless we returned to it: there was nowhere else to go. We returned to New York, appropriately-to the homeland of the uprooted, where everyone you met came from another town and tried to forget it; where nobody seemed to have parents, or a past more distant than last night's swell party, or a future beyond the swell party this evening and the disillusioned book he would write tomorrow" (Cowley 1969: 47).

6 El mito de la Tierra Baldía aparece por primera vez en el romance francés medieval Perceval o el Cuento del Grial de Chrétien de Troyes, compuesto, parece, a finales del siglo XII, en torno al año 1180. En dicho romance, el joven caballero Perceval visita el castillo del Rey Pescador, un rey herido en los muslos cuya impotencia sexual trae consigo la infertilidad de su reino. En esta primera versión, la restauración de la fertilidad de la tierra, así como la curación del rey, sólo se producirá si el joven caballero pregunta a quién sirve el Grial que aquél contempla en el castillo del Rey Pescador. Versiones posteriores del mito incorporan la búsqueda de este (Santo) Grial para lograr la restauración de la Tierra Baldía y la
} 
la explicación de T. S. Eliot sobre cómo el método mítico constituye la única herramienta útil para ordenar y dar significado al mundo moderno, el cual funciona de forma caótica, anárquica y fútil (vid. Eliot 1942). Desde esta perspectiva, tal y como se explicará más adelante, puede argumentarse que, en efecto, la novela de Dos Passos plantea la reinterpretación de la ciudad moderna como el correlato modernista de la Tierra Baldía mítica del ciclo artúrico. Así, puede entenderse que la gran ciudad moderna -una isla de hormigón y acero rodeada de agua "rota" (Dos Passos 2000: 15), estancada y contaminada- constituye en realidad un espacio mítico ${ }^{7}$ marcado por la podredumbre, la desolación y la enfermedad. A colación de este argumento, debe tenerse en cuenta la explicación que aporta Leslie Fiedler sobre los procesos de mitificación del espacio urbano: "It [la ciudad] has proved oddly resistant to any mythic images except for certain negative, dark, infernal ones, which reinforce rather than neutralize our sense of alienation" (Fielder 1981: 114). Tal mitificación negativa, si se permite la expresión, resulta especialmente profusa entre los contemporáneos de T. S. Eliot, así como entre sus predecesores y sucesores más inmediatos (Fielder 1981: 115).

Manhattan Transfer comienza con la siguiente descripción:

Three gulls wheel above the broken boxes, orangerinds, spoiled cabbage heads that heave between the splintered plank walls, the green waves spume under the round bow as the ferry, skidding on the tide, crashes, gulps the broken water, slides, settles slowly into the slip. (Dos Passos 2000: 15)

El espacio urbano acumula desechos y funciona, desde el principio de la novela, como prolongación semántica de una comunidad estigmatizada, de una parte, por la esterilidad moral y espiritual, y, de otra, por la frustración vital de los individuos que la conforman. Si el espacio de la ciudad se origina y se desarrolla a partir de las estructuras culturales que acoge, es fácil comprender que la representación literaria de dicho espacio funcione como reflejo de la condición moral, espiritual y estética de la comunidad que habita el espacio urbano. Ocurre así en Manhattan Transfer. Los habitantes de la ciudad, todos ellos enfermos, viven marcados por la esterilidad, la frustración y el colapso moral. En consecuencia, la representación del espacio urbano en la novela se configura, desde el

sanación del rey mítico. La relevancia de este mito en la literatura modernista en lengua inglesa no puede desligarse de la influencia de The Waste Land (La tierra baldía, 1922), de T. S. Eliot. En relación con esto, vid. Lowry (1974).

7 Tal afirmación coincide, una vez más, con la noción de 'heterotopía', entendida ésta como "una suerte de contestación a un tiempo mítica y real del espacio en que vivimos" (Foucault 1997: par. 14; mi cursiva). 
paradigma propuesto por Scott y Simpson-Housley, como "la amenaza de Babilonia" (Scott y Simpson-Housley 1994: 335). Scott y Simpson-Housley argumentan: "In western letters, then, urban realties are located over against the assumed innocence of an Eden now lost, but somehow promised again in a New Jerusalem, which itself may only be understood in the contrast of a sinful Babylon" (Scott y Simpson-Housley 1994: 331). La representación de la ciudad como 'nueva Babilonia', por así decirlo, constituye pues una amenaza. Esta amenaza se configura como sigue: "the threat of Babylon occurs in various images of alienation, expressed in a spectrum of suffering ranging from homelessness through gender differences to urban violence, even impending, economic, social and political chaos" (Scott y Simpson-Housley 1994: 335). Tal es el caso de Manhattan Transfer. Arrington explica:

In the Third Section of John Dos Passos's Manhattan Transfer, both the opening and closing chapter titles are direct quotations from two rather obscure Old Testament prophets. 'Rejoicing City That Dwelt Carelessly' comes from Zephaniah [...] [and] the title of the concluding chapter ['The Burthen of Nineveh'] borrows from the opening line of Nahum [...] Jeremiahs both, these prophets sing of the destruction of Judah, Nineveh, and Babylon, cities corrupted by their own wealth and success; and Dos Passos remembers their laments in warning New York, and America of a similar fate. Dos Passos had made this comparison between New York and its biblical counterparts several years before Manhattan Transfer appeared. In a letter to Germaine Lucas-Chamionniere, he admits that New York, for all its magnificence, reminded him of 'Nineveh and Babylon, of the Ur of Chaldees, of the immense cities which loom like basilisks behind the horizon in ancient Jewish tales, where the temples rose as high as mountains and people ran trembling through dirty little alleys to the constant noise of whips with hilts of gold'. (Arrington 1982: 438)

La identificación entre Manhattan y Babilonia en el capítulo titulado "Rejoicing City that Dwelt Carelessly"8 se torna catastrófica, pues dicho capítulo comienza con el siguiente epígrafe: "In the subway their eyes pop as they spell out APOCALYPSE, typhus, cholera, shrapnel, insurrection, death in fire, death in water, death in hunger, death in mud" (Dos Passos 2000: 247). Así comienza la tercera (y última) sección de la novela. Se combina por tanto el colapso moral de la 'nueva Babilonia' con la retórica de la

\footnotetext{
${ }^{8}$ Nótese, por cierto, a colación del descuido como nexo de unión comunitario, las siguientes líneas de The Great Gatsby (1925): "They were careless people, Tom and Daisy-they smashed up things and creatures and then retreated back into their money or their vast carelessness, or whatever it was that kept them together, and let other people clean up the mess they had made [...]" (Fitzgerald 1991: 147; mi cursiva).
} 
enfermedad, que recrea Nueva York como un espacio plagado por la muerte (en el fuego, en el agua, en el barro, por la metralla, de hambre) y la enfermedad (tifus, cólera) que, en realidad, constituye la metáfora perfecta de una condición espiritual de degeneración que afecta al conjunto de la comunidad como si de una pandemia se tratase. En última instancia, el espacio urbano -contagiado de la afección comunitaria- se configura de manera simbólica como responsable de dicha pandemia - 0 , al menos, se percibe como tal. Malcolm Bradbury explica: "Manhattan [is] a vast collective motion, a mechanical womb, a machine for living and suppressing life [...] [so] what is displaced from the individual life is reinvested in the operational city itself. Mechanism and destructiveness are dominant, and characters become like the impersonal environment through which they move" (Bradbury 1992: 107). La ciudad priva a sus ciudadanos de toda energía vital. Los procesa, como una máquina trituradora de alimentos. El epígrafe que abre la novela concluye así: "Handwiches whirl with jingle of chains. Gates fold upwards, feet step out across the crack, men and women press through the manuresmelling wooden tunnel of the ferry-house, crushed and jostling like apples fed down a chute into a press" (Dos Passos 2000: 15; mi cursiva). La primera imagen de la novela describe la ciudad como una máquina que aplasta a los individuos 9 . De manera muy significativa, el nacimiento de Ellen se narra inmediatamente después. Y no se olvide que las aspiraciones vitales de la protagonista (si es que puede hablarse de protagonistas individuales en Manhattan Transfer) serán aplastadas "like sausage meat" (Dos Passos 2000: 115 ) en el transcurso de la novela.

Como se ha apuntado, el espacio urbano constituye la prolongación semántica de la masa urbana que lo habita. Así, por cuanto dicha masa está enferma, el propio espacio se representa como afectado por la misma enfermedad que padece la comunidad urbana. De este modo, el espacio urbano enfermo constituye el correlato simbólico de una comunidad marcada por la degeneración y la frustración. En este sentido, Richard Lehan explica: "Dos Passos's novel conveys a Freudian truth [...]. Civilized life inhibit[s] hostility, driving it inward; the city, aligned with the superego, restrain[s] the vitality of the psyche. Life in the metropolis, as both Dos Passos and Freud saw it, thus became more neurotic" (Lehan 1998: 238). En efecto, según Freud, "el término cultura designa la suma de las producciones e instituciones que distancian nuestra vida de la de

\footnotetext{
${ }^{9}$ Esta imagen se retomará más adelante, cuando Jimmy rechace la oportunidad de trabajar para su tío por temor a que su vida sea procesada como la carne de las salchichas: "Jimmy fed in a tape in and out the revolving doors, noon and night and morning, the revolving doors grinding out his years like sausage meat" (Dos Passos 2000: 115).
} 
nuestros antecesores animales y que sirven a dos fines: proteger al hombre contra la Naturaleza y regular las relaciones de los hombres entre sí" (Freud 1999: 35). Por cuanto la ciudad acoge dichas instituciones y articula las relaciones entre individuos, puede argumentarse que el espacio urbano constituye la representación espacial de la cultura. Desde una perspectiva freudiana, tal representación sólo puede encarnarse retóricamente desde el campo antropológico de la enfermedad, pues "la cultura reposa sobre la renuncia a las satisfacciones instintuales". Se genera por tanto una "frustración cultural" (Freud 1999: 43) que se manifiesta en "la miseria psicológica de las masas" (Freud 1999: 60). Así, el espacio de la ciudad funciona como metáfora de una enfermedad colectiva que es, en realidad, consecuencia de la cultura o, mejor, de las imposiciones del "super-yo cultural" (Freud 1999: 87). De manera ineluctable, la mencionada "frustración cultural" deviene en neurosis, esto es, en una patología colectiva: "el ser humano cae en la neurosis porque no lograr soportar el grado de frustración que le impone la sociedad en aras de sus ideales de cultura" (Freud 1999: 32).

Nótese entonces cómo todos los personajes de Manhattan Transfer están marcados por la frustración. Primero, el espacio urbano se configura como emblema del malestar de la comunidad. Segundo, este malestar comunitario se manifiesta, sobre todo, en la frustración vital de Jimmy y de Ellen, los personajes principales. Así, la ciudad misma funciona como metáfora de una enfermedad que, de manera irremediable, consume la vida de quienes habitan el espacio urbano. Según Eric Mottram, Nueva York es "una máquina para vivir la muerte" (Mottram 1971: 240). Tal afirmación sugiere una paradoja curiosa y muy elocuente, pues la noción de vivir la muerte lleva a considerar que la aparente vida es, en realidad, un estado de muerte. Así pues, puede incluso argumentarse que la "ciudad histérica" de Adams se ha transformado, en los años veinte, en una ciudad muerta. Los ciudadanos de Manhattan ya no viven la vida, sino que viven la muerte. O, mejor, los personajes de Manhattan Transfer viven un simulacro de vida, esto es, una vida reconstruida artificialmente y sometida a unas imposiciones culturales que sólo pueden desencadenar una neurosis generalizada. Todos los personajes sienten una profunda aversión vital. Todos están ineludiblemente abocados a la muerte, bien se entienda ésta en un sentido literal, bien se identifique con la esterilidad, la enfermedad o al frustración vital. Los personajes Jimmy y Ellen, cuyas vivencias funcionan a modo de nexo de unión entre las docenas de historias y personajes que se yuxtaponen en la novela, constituyen los ejemplos más ilustrativos de cómo es posible vivir la muerte o, mejor dicho, cómo es posible vivir en ausencia de vida.

El proceso vital de Ellen que describe la novela -ésta se abre con el nacimiento de Ellen $\mathrm{y}$, puede argüirse, se cierra con su 
muerte- es de hecho un proceso de muerte. La transformación vital de Ellen es en realidad un proceso de cristalización, similar al de una flor que se seca para evitar marchitarse y que, en consecuencia, permanece eternamente en un estado de muerte en vida. Siendo aún una niña, ella misma decide cambiarse el nombre. Ellen deja de llamarse Ellen para convertirse en "Elaine the lilly maid of Astalot" (Dos Passos 2000: 58). La referencia a la dama de Astolat anticipa el destino de frustración que aguarda al personaje ${ }^{10}$. Ellen se ha convertido en Elaine, y, más tarde, cuando se complete su proceso de frustración vital, se convertirá en Helena. En el ciclo artúrico, la frustración de los deseos de Elaine de Astolat trae consigo su muerte. La frustración de los deseos de Ellen, de manera simbólica, también ocasiona la muerte -esto es, la muerte en vida- del personaje.

Ellen, como Elaine de Astolat y Lucía de Lammermoor $^{11}$, también desea casarse. Sin embargo, la fatalidad del personaje consiste precisamente en una sucesión de matrimonios que no acaba por satisfacer las aspiraciones ideales de su imaginación. Todo lo contrario, las frustra. La muerte simbólica (pero definitiva) de Ellen acontece cuando finalmente acepta casarse con George Baldwin, el cual había deseado a aquélla desde el comienzo de la novela ${ }^{12}$. Aún así, la frustración vital del personaje se inicia con su primer matrimonio. Siendo aún muy joven, Ellen se casa con John Oglethorpe, un productor de espectáculos de variedades homosexual. Ellen proyecta sus sueños infantiles en el matrimonio, esto es, en la institución social que, en la cultura, viene a sustituir a un rito de fertilidad. Así, cuando los deseos de Ellen se hacen realidad, la materialización de sus aspiraciones produce en ella un rechazo manifiesto por la vida:

${ }^{10}$ Elaine de Astolat es un personaje de la leyenda artúrica, cuyo destino trágico viene determinado por el amor no correspondido que siente por Lancelot: "I take God to my record I loved never none but Sir Launcelot Du Lake, not ever shall, and a clean maiden I am for him and for all other; and sithen it is the sufferance of God that I shall die for the love of so noble a knight [...] good Lord, I might not withstand the fervent love wherefore I have my death" (Malory 1969, II: 413). Cuando Ellen pide a Alice que no la vuelva a llamar 'Ellen', Alice responde: "Well Elaine then, Elaine the lily maid of Astalot" (Dos Passos 2000: 59). Se trata de una referencia directa al poema de Alfred, Lord Tennyson, "Lancelot and Elaine" (v. 2), incluido en Idylls of the King -un ciclo de doce poemas narrativos de tema artúrico publicados entre 1856 y 1885-. Dicho esto, debe tenerse en cuenta que Alice pronuncia mal el nombre del personaje, confundiendo "Astolat" (en Tennyson) con "Astalot".

${ }^{11}$ Se trata de la protagonista de The Bride of Lammermoor, una novela histórica de Walter Scott publicada en 1819. La novela narra la trágica historia de amor entre Lucy Ashton y Edgar Ravenswood, enemigo de la familia de ella.

${ }^{12}$ Baldwin va a convertirse en alcalde de Nueva York. El personaje es por tanto emblema de la propia ciudad. 
The rain lashed in her face spitefully stinging her flesh, wetting her nightdress. She pushed her forehead against the frame. Oh I want to die. I want to die. All the tight coldness of her body was clenching in her stomach. Oh I'm going to be sick. She went into the bathroom and closed the door. When she had vomited she felt better. Then she climbed into bed again careful not to touch John. If she touched him she would die. (Dos Passos 2000: 112)

Este pasaje describe la noche de bodas de Ellen y Oglethorpe. Ellen es, literalmente, "the Bride of Lammermoor", pero también "Elaine of Astolat", pues su frustración implica de modo inexorable el deseo de morir. El matrimonio -el rito de fertilidad transformado en institución cultural- resulta frustrado hasta el extremo de producir en Ellen síntomas físicos de enfermedad, pues llega a sentir náuseas al verse atrapada en la institución: "Oh I'm going to be sick. She went into the bathroom and closed the door. When she had vomited she felt better" (Dos Passos 2000: 112). Con la frente apoyada en la ventana, sobre la que resbala la lluvia, Ellen se hace consciente de su propia enfermedad: "Oh I'm going to be sick" (Dos Passos 2000: 112). Por supuesto, la enfermedad de Ellen debe leerse como la manifestación individual de una patología colectiva. Además, debe tenerse en cuenta que, hasta tal punto participa el espacio urbano (y sus instituciones) de la enfermedad de la masa que, en determinadas ocasiones, los habitantes de la ciudad perciben a ésta como responsable de su frustración individual -quizá, por cuanto la ciudad constituye la manifestación espacial de la cultura. Nótese la siguiente escena clave de la novela:

When he stretched his legs out under the table he touched her feet. She drew them away. Jimmy could feel his jaws chewing, they clanked so loud under his cheeks he thought Ellie must hear them. She sat opposite of him in a gray tailoredsuit, her neck curving up heartbreakingly from the ivory $\mathrm{V}$ left by the crisp frilled collar of her blouse, her head tilted under tight gray hat, her lips made up; cutting up little pieces of meat and not eating them, not saying a word. (Dos Passos 2000: 272; mi cursiva)

Jimmy y Ellen cenan en un restaurante clandestino que sirve alcohol ilegalmente ${ }^{13}$. Jimmy observa a Ellen. Ésta trocea la carne pero no la prueba. Él la toca. Ella se aparta. Él escucha el sonido de sus propias mandíbulas, mientras fija su mirada en la $\mathrm{V}$ que estiliza el cuello de Ellen. Las percepciones sensoriales se acumulan mientras Ellen, sentada justo a su lado, paradójicamente se aleja cada vez

13 La 'Ley Seca', resultado de la decimoctava enmienda de la constitución, estuvo en vigor entre el período de 1920 y 1933. 
más. Finalmente, casi de milagro, Jimmy huele a Ellen. Se desencadena la epifanía:

He felt paralyzed like in a nightmare; she was a porcelain figure under a bellglass. A current of fresh snowrinsed air from somewhere eddied all of a sudden through the blurred packed jangling glare of the restaurant, cut the reek of food and drink and tobacco. For an instant he caught the smell of her hair. The cocktails burned in him. God I don't want to pass out.

Sitting in the restaurant of the Gare de Lyon, side by side on the black leather bench. His cheek brushes hers when he reaches to put herring, butter, sardines, anchovies, sausage on her plate. They ate in a hurry, globbing, giggling, gulp wine, start at every screech on an engine $[\ldots]$ (Dos Passos 2000: 272)

La conciencia de Jimmy se traslada a Francia, a una noche que pasaron Ellen y él en el transcurso de una travesía ferroviaria a través del país. El contraste entre ambas escenas es bastante elocuente. Fuera de Nueva York, los personajes comen con avidez; Jimmy sirve la comida a Ellen y sus mejillas se rozan. Ella no se aparta. La oposición simbólica entre los espacios cerrados de la ciudad y el tren, un espacio móvil que recorre en una noche las distintas regiones de Francia, se exacerba cuando Jimmy recuerda:

Side by side in the window in the lurching jiggling corridor. Deedleddump, going south. Gasp of nightingales along the track among the silverdripping poplars. The insane cloudy night of moon light smells of gardens garlic rivers freshdunged field roses. Gasp of nightingales. Opposite of him the Elliedoll was speaking. (Dos Passos 2000: 273)

Las reconstrucciones artificiales de las flores -esto es, las flores pintadas, cosidas o tapizadas- constituyen, a lo largo de la novela, el emblema de la frustración vital de Ellen ${ }^{14}$. El olor de las rosas del campo en el pasaje anterior constituye el opuesto simbólico de las

${ }^{14}$ Ellen parece mantener cierta conexión con el símbolo de las flores muertas desde el principio de la novela: "stepping carefully among the roses on the sunny field of the carpet, little Ellen danced" (Dos Passos 2000: 28). La misma alfombra estampada con rosas vuelve a aparecer años más tarde, en la visita de Ellen a su padre (el señor Thatcher) para anunciar su divorcio de Oglethorpe: "Ellen dropped onto the Davenport and let he eyes wander among the faded red roses of the carpet" (Dos Passos 2000: 183). Ellen siente atracción por las flores artificiales flores que nacen y viven muertas para siempre, como una muñeca de porcelana en un tarro de cristal (Dos Passos 2000: 273)- porque Ellen siente aversión por la vida: "Laying her gloves away on the edge of the table her hand brushed against the base of rusty red and yellow roses. A shower of faded petals fluttered onto her hand, her gloves, the table. She shook them off with her hands. 'And do have him take these wretched roses away George... I hate faded flowers'" (Dos Passos 2000: 200). 
flores artificiales y, por tanto, da cuenta de un espacio alternativo a la ciudad donde las flores silvestres sí pueden crecer. Jimmy experimenta la epifanía -el olor de las rosas- a partir del olor del cabello de "Elliedoll", quien ya no es una joven flor -"the lilly maid of Astalot" (Dos Passos 2000: 58; mi cursiva)-, sino "a porcelaine figure under a bellglass" (Dos Passos 2000: 272):

Opposite of him the Elliedoll was speaking. 'He says the lobster salad's all out... Isn't that discouraging?'

Suddenly he had his tongue. 'Gosh if that were the only thing.'

'What do you mean?'

'Why did we come back to this rotten town anyway?' (Dos Passos 2000: 273)

Lo primero que Jimmy percibe en el instante en que su conciencia vuelve a Manhattan es el proceso de cristalización de Ellen: "Opposite of him the Elliedoll was speaking" (Dos Passos 2000: 273). De inmediato, Jimmy culpa a la "ciudad podrida", Nueva York, donde Ellen y él han vuelto tras pasar unos años en Europa durante la Primera Guerra Mundial": "Why did we come back to this rotten town anyway?" (Dos Passos 2000: 273) ${ }^{15}$. Desde la perspectiva de Jimmy, Manhattan es responsable de su propia frustración vital, así como de la frigidez de Ellen, la cual aparta los pies al contacto con la piel del personaje masculino. Por el contrario, Europa se percibe como un espacio de sosiego y plenitud vital en el que pueden darse escenas pastoriles que configuran un espacio edénico -esto es, mítico- por oposición al espacio urbano de Nueva York:

Before the kid was born Ellie sometimes had toobright eyes like that. The time of the hill when she had suddenly wilted in his arms and been sick, and he had left her among the munching, calmly staring cows on the grassy slope and gone to a shepherd's hut and brought back milk in a wooden ladle, and slowly as the mountains hunched up with evening the color had come back into her cheeks and she had looked at him that way and said with a dry little laugh: It's the little Herf inside me. (Dos Passos 2000: 290)

\footnotetext{
${ }^{15}$ Resulta significativo que Jimmy comprenda la fuerza perversa y corruptora de la ciudad tras su vuelta de Europa. Nótese la explicación que Malcom Cowley ofrece acerca de la impresión que la vuelta a Nueva York ejercía sobre los exiliados en Europa -entre ellos, John Dos Passos-: "New York, to one returning from Paris or London, seems the least human of all the babylons. Its life is expressed in terms of geometry and mechanics: the height and cubical content of its buildings, the pressure that squeezes them upward like clay squeezed out between the fingers, the suction that empties one district to overcrowd another, the lines of force radiating from subway stations, the density of traffic. Its people have a purely numerical function: they are counted as units that daily pass a given point. Their emotions are coefficients used in calculating the probability of trade" (Cowley 1969: 201).
} 
Como puede observarse, todo espacio que se sitúa fuera de Manhattan se percibe como un espacio idílico, fértil y pacífico, un locus amoenus en el que los personajes pueden escapar de la esterilidad (Ellen está embarazada) y la frustración inherentes a sus vidas en la ciudad. De manera muy elocuente, el pasaje anteriormente citado describe a Ellen enferma, pero el espacio idílico no sólo no participa de tal enfermedad, sino que además propicia la curación del personaje. Por oposición, el espacio urbano se configura como un espacio mítico perverso. Dicha mitificación perversa permite interpretar la representación de la ciudad en Manhattan Transfer como reinterpretación modernista el mito pre-moderno de la Tierra Baldía. Por consiguiente, a la hora de considerar la realidad o irrealidad del espacio urbano recreado en la novela, debe tenerse en consideración la intertextualidad ${ }^{16}$ de The Waste Land (1922), de T. S. Eliot. Así, la representación de Manhattan en el texto de Dos Passos debe entenderse -por cuanto es también correlato de la Tierra Baldía mítica- como recreación narrativa de la "ciudad irreal" eliotiana -el espacio modernista de muerte y enfermedad por excelencia: "Unreal city / Under the brown fog of a winter dawn / A croad flowed over London Bridge, so many, / I had not thought death had undone so many" (Eliot 2001: vv. 60-63).

En Manhattan Transfer, por cuanto el espacio articula un determinado orden social, aquél exacerba la fuerza destructora que las instituciones comunitarias ejercen sobre los individuos. He aquí el origen de la enfermedad comunitaria. Sam See explica:

In point of fact, throughout the text, groups of characters are typically mentioned as a homogonous mass [...] or as impersonal synecdoches [...]. The text's narrative voice (which varies wildly, including third limited, second person, and free indirect discourse) compounds this homogeny via, paradoxically, heterogeneity, for no single narrative voice claims authority. (See 2008: 351)

El propio discurso narrativo disuelve así a los individuos en una masa homogénea, tal y como hace la ciudad ${ }^{17}$. Obsérvese la percepción que tiene Jimmy del vestíbulo de un edificio de oficinas: "softcheeked girls chewing gum, hatchetfaced girls with bangs, creamfaced boys his own age, young toughs with their hats on one side, sweatyfaced messengers, criss-cross glances, sauntering hips, red jowls masticating cigars, sallow bodies of elderly men" (Dos

16 En este contexto, la noción de intertextualidad debe entenderse como "la presencia efectiva de un texto en otro" (Genette 1989: 10).

17 Sobre le estructura narrativa de Manhattan Transfer, la yuxtaposición como técnica y la "hermenéutica de la simultaneidad" en su relación con la geografía del espacio urbano, vid. See (2008). 
Passos 2000: 115). La descripción de Jimmy refleja una disociación progresiva: comienza diluyendo a los individuos en grupos estereotipados y culmina con la desintegración de aquellos en meras partes corporales. Según explica Gelfant, "as [the characters] move through the city they perceived people and objects as dissociated images - that is, they do not receive unified and total impressions of entities but only fragmentary impressions of parts of the whole" (Gelfant 1974: 47). La ciudad disocia la individualidad de los personajes; sólo cuando al final Jimmy abandona Manhattan puede aferrarse de nuevo a una voluntad individual que ha tratado inútilmente de preservar a lo largo de la novela: "the only passenger on the ferry, he roams round as if he owned it" (Dos Passos 2000: 359). La disolución de la individualidad -paralela a la "frustración cultural" (Freud 1999: 43) inherente a la vida urbana- tiene como resultado una "sociedad atomística" (Vanderwerken 1977: 254), que habita "la Ciudad de la Destrucción" (Vanderwerken 1977: 256).

Según argumenta este artículo, la frustración (y la disolución) del individuo se expresa en el texto a través de la retórica de la enfermedad. Sin embargo, como ya se ha adelantado, dicha retórica no posee únicamente un valor simbólico ${ }^{18}$, sino que adquiere dimensiones míticas. Manhattan Transfer reinterpreta el mito medieval de la Tierra Baldía, para así dar cuenta de la concepción modernista del espacio urbano, es decir, la concepción de la ciudad moderna como un espacio marcado por la enfermedad, la esterilidad y la degeneración. Desde un punto de vista mitocrítico, debe reseñarse que la reinterpretación de mito de la Tierra Baldía articulada en el texto constituye una reinterpretación no ya pesimista, sino, en cierto modo, apocalíptica. Las estructuras sociales articuladas por el espacio urbano no contemplan la figura de un individuo rector en el cual tengan origen las distintas relaciones comunitarias ${ }^{19}$. En la ciudad no hay un rey enfermo, como en el mito original, pues en la ciudad no hay rey posible. En cierto sentido, todos los ciudadanos son el rey, pues todos supeditan su individualidad a la constitución de una entidad uniforme y emblemática: la masa urbana (enferma). Así, la relación espiritual inextricable entre rey $y$ reino que subyace al mito medieval se

\footnotetext{
${ }^{18}$ It [la ciudad en la literatura] seems to function primarily as both an emblem and an archetype. As such it has more various and more diffuse associations and resonances than a symbol can generally encompass" (Pike 1981: 13).

19 Para poder llevar a cabo un estudio del mito de la Tierra Baldía en profundidad, es preciso entender, primero, que la narración medieval reproduce simbólicamente el funcionamiento de la monarquía, esto es, la principal institución política medieval, por cuanto el citado mito da cuenta de una relación mística inextricable entre la salud del monarca y la prosperidad del reino. Tal fundamentación sociopolítica del mito no ha lugar en un proceso de mitificación del espacio urbano contemporáneo.
} 
transforma en una relación mística entre espacio y comunidad, de manera que la representación simbólica de una masa urbana enferma no puede sino traer consigo la representación de un espacio urbano también enfermo. En la ciudad no hay ciudadanos privados. La masa es la ciudad misma, por cuanto la masa configura las distintas estructuras sociales e instituciones comunitarias, cuya articulación espacial es, precisamente, la ciudad.

Como en el mito pre-moderno referido, el principal síntoma de la enfermedad comunitaria es la esterilidad. No obstante, la enfermedad de la ciudad no obedece a una causa externa, como ocurre en el mito medieval, y, por tanto, no puede ser sanada. La ciudad está enferma y es estéril, por cuanto es ciudad. Spengler escribe: "La civilización trae consigo la crisis de la fecundidad. Las raíces antiquísimas de la existencia se secan en los adoquines de las ciudades" (Spengler 1966: 114) ${ }^{20}$. En efecto, esta hipótesis se manifiesta en Manhattan Transfer. En consonancia con el argumento de Spengler, en la novela de Dos Passos "la ciudad niega toda naturaleza [...]. La ciudad inventa una naturaleza artificial, pone fuentes en lugar de manantiales, cuadros de flores, estanques, tallos recortados, en lugar de praderas, charcas y matorrales" (Spengler 1966: 116-117). Manhattan Transfer acumula alfombras, cortinas y estampados floridos ${ }^{21}$. En la gran ciudad, pues, la cultura -acaso la muerte de la cultura- sustituye a la naturaleza. Para Bradbury, ésta es la principal característica de la ciudad moderna: "The modern city has appropriated most of the functions and communications of society, most of its population, and the furthest extremities of its technological, commercial, industrial and intellectual experience. The city has become culture, or perhaps the chaos that succeeds it" (Bradbury 1991: 97). Spengler coincide con esta última idea. Para el filósofo alemán, las consecuencias que implica la reconstrucción artificial de la vida son catastróficas, pues "el coloso pétreo de la ciudad mundial señala el término del ciclo vital de toda gran cultura" (Spengler 1966: 122). Desde esta perspectiva, el espacio urbano moderno da cuenta de una comunidad no ya enferma, sino moribunda. La novela de Dos Passos parece corroborar esta noción recuérdese: Manhattan es "una máquina para vivir la muerte" (Mottram 1971: 240; mi cursiva) - a través, precisamente, de la reinterpretación morbosa de símbolos tradicionales de regeneración

\footnotetext{
${ }^{20}$ La Decadencia de Occidente de Spengler se traduce al inglés en 1922, el mismo año en que se publica The Waste Land -esto es, el texto que activa la reinterpretación modernista del mito medieval de la Tierra Baldía-. La influencia de ambos textos se combina en Manhattan Transfer para representar la ciudad moderna como un espacio de enfermedad.

${ }^{21}$ Vid. sup. nota 12.
} 
como el agua, el fuego o las flores ${ }^{22}$, así como del cuestionamiento de las estructuras míticas tradicionales. En Manhattan Transfer, el ciclo vital de la naturaleza, esto es, la regeneración primaveral de la Tierra Baldía, se ve truncado. Al término de la novela Jimmy abandona la ciudad junto con un camión cargado de flores vivas: "A rich smell of maytime earth comes from it, of wet flowerpots and greenhouses" (Dos Passos 2000: 359). La primavera -las flores que crecen en "la tierra de mayo"- debe mudarse fuera de la ciudad para poder sobrevivir.

Para Spengler, "el acceso de Nueva York al campo de ciudad mundial, tras la guerra de Secesión de 1861-1865, es acaso el acontecimiento del pasado siglo más fecundo en consecuencias" (Spengler 1966: 122). Las consecuencias a las cuales se refiere son, por supuesto, calamitosas:

La existencia pierdes sus raíces y [...] de este hecho, empero, se deriva un fenómeno que viene desde hace tiempo iniciándose en silencio y que ahora entra de pronto en la luz cruda de la historia, para preparar el desenlace de todo el drama. Me refiero a la infecundidad del hombre civilizado [...]. Trátase, ni más ni menos, de una propensión metafísica a la muerte. El último hombre de la gran urbe no quiere vivir, se aparta de la vida, no como individuo, pero sí como tipo, como masa. (Splenger 1966: 127)

Una vez el individuo es absorbido por la masa, su pulsión vital desaparece. Así le ocurre a Ellen, quien "embraces sterility as her destiny" (Gelfant 1974: 52) y, por tanto, constituye un ejemplo muy ilustrativo de la "infecundidad del hombre civilizado" que menciona Spengler. Nótese cómo, una vez más, el principal síntoma de la enfermedad comunitaria se identifica con la esterilidad, esto es, la piedra de toque del mito de la Tierra Baldía. Sin embargo, tal esterilidad se produce por cuanto el individuo se disuelve en la masa urbana. Sólo como individuo puede el ciudadano liberarse de esa "propensión metafísica a la muerte" (Splenger 1966: 127), la cual se representa en el texto mediante la retórica de la enfermedad -una enfermedad que, como una plaga, afecta al conjunto de la masa urbana. Por este motivo sólo Jimmy, solo, aislado, consigue abandonar la ciudad, así como la infecundidad inherente al espacio urbano. Al final de la novela, Jimmy es "Longlegged Jack of the Isthmus" (Dos Passos 2000: 113), el único superviviente del diluvio:

22 Los símbolos funcionales más elocuentes de la novela son el coche de bomberos (esto es, un significante que indica fuego y cuya presencia, en todos los casos, connota bien muerte, bien peligro) y las flores artificiales. El coche de bomberos está asociado emblemáticamente al personaje de Jimmy, mientras que las flores muertas son el símbolo asociado a Ellen. Tanto el coche de bomberos como las flores artificiales son significantes de la aversión por la vida (y la pulsión de muerte) que sienten ambos personajes. 
"he walks on, taking pleasure in breathing, in the beat of his blood, in the tread of his feet on the pavement" (Dos Passos 2000: 360).

Como se ha venido argumentando, la ciudad de la novela no es una reproducción realista de Nueva York a comienzos del siglo XX. El espacio urbano del modernismo se aparta de los patrones naturalistas - "Manhattan Transfer is no more 'naturalistic' than The Waste Land" (Lowry 1974: 60)-. Las ciudades del modernismo, como ya se explicó al comienzo del artículo, son "metaphor rather than place" (Bradbury 1991: 97). Entonces, en la novela de Dos Passos, Manhattan se configura como metáfora de una forma de vida mecanizada, marcada por la alienación y la esterilidad moral y espiritual. La ciudad de la novela funciona pues como un espacio mítico de enfermedad, cuyo significado trasciende las coordenadas históricas y geográficas de Nueva York.

En su comentario a Manhattan Transfer, Alfred Kazin escribe: "The book was like a perverse esthetic geometry in which all the colors of the city's scenes were daubed together madly, and all its framed jumbled. What one saw in Manhattan Transfer was not the broad city pattern at all, but a wistful absorption in monstrousness" (Kazin 1995: 350). Desde este punto de vista, cabe entonces interpretar que Manhattan en la novela constituye, en efecto, un espacio mítico monstruoso. La Tierra Baldía ya no es el suelo mítico donde tiene lugar la regeneración primaveral y la curación del Rey Pescador. Por el contrario, la Tierra Baldía "urbana" constituye un paisaje apocalíptico, en el cual la enfermedad está tan extendida que no puede concebirse curación posible. Recuérdese: "In the subway their eyes pop as they spell out APOCALYPSE, typhus, cholera, shrapnel, insurrection, death in fire, death in water, death in hunger, death in mud" (Dos Passos 2000: 247). La novela está plagada de muerte pero, sobre todo, está infestada de lugares y personajes enfermos ("tifus, cólera"), destinados en la mayoría de los casos a una muerte por agua o por fuego, las cuales, precisamente, dan cuenta de la imposibilidad de regeneración. Tal imposibilidad de regeneración afecta al espacio urbano y a los ciudadanos. Éstos y aquél son víctimas de una plaga que, en última instancia, padece cada elemento constitutivo de la ciudad moderna. Desde la perspectiva del texto, la esterilidad tanto emocional como espiritual de los ciudadanos es consecuencia de un determinado orden social y de una coyuntura histórica, de los cuales, precisamente, la ciudad moderna es emblema. Así pues, la ciudad se configura en el texto como un espacio mítico de enfermedad, degeneración y deshumanización que, desde el conjunto de significados de la novela, se intuyen irreversibles. En consecuencia, puede argüirse que, primero, Manhattan Transfer reproduce y reinterpreta el mito premoderno de la Tierra Baldía -es decir, un mito de enfermedad y regeneración-; y, segundo, la novela de Dos Passos, a través de un 
proceso de mitificación morbosa, configura el espacio urbano como metáfora de una afección social que estigmatiza a la comunidad moderna. Desde los códigos simbólicos del texto, la degeneración de dicha comunidad -moderna y, por tanto, urbana- se intuye irreparable.

\section{Bibliografía}

ADAMS, Henry (1961): The Education of Henry Adams. An Autobiography. Boston: Houghton Mifflin Company / The Riverside P.

ARRINGTON, Phillip (1982): "The sense of an ending in Manhattan Transfer" [en línea]. American Literature, 54.3, pp. 438-443. En: JSTOR (http://www.jstor.org/stable/2925854) [Consulta: 13/10/2010].

BRADBURY, Malcom (1991): "The cities of Modernism", en Malcolm Bradbury y James McFarlane (eds.), Modernism 1890-1930, pp. 96103. London: Penguin Books.

- (1992): The Modern American Novel. Oxford: OUP.

CAROL OATS, Joyce (1981): "Imaginary Cities: American", en Michael C. Jaye y Ann Chalmers Watts (eds.), Literature and the American Urban experience. Essays on the City and Literature, pp. 11-33. Manchester: Manchester University Press.

COWLEY, Malcom (1969): Exile's Return: a Literary Odyssey of the 1920s. New York: The Viking Press.

DOS PASSOS, John (2000): Manhattan Transfer. London: Penguin Books.

ELIOT, T. S. (1942): "Ulysses, Order and Myth", en John W. Aldridge (ed.), Critiques and Essays on Modern Fiction. 1920-1951. Representing the Achievement of Modern American and British Critics, pp. 424-426. New York: The Roland P. Company.

- (2001): The Waste Land. Authoritative Text, Contexts and Criticism. Michael North, ed. London, New York: Norton \& Company.

FIELDER, Leslie (1981): "Mythicizing the City", en Michael C. Jaye y Ann Chalmers Watts (eds.), Literature and the American Urban experience. Essays on the City and Literature, pp. 113-129. Manchester: Manchester University Press.

FITZGERALD, Francis S. (1991): The Great Gatsby. London: Longman Group Limited.

FOUCAULT, Michael (1997): "Los espacios otros" [en línea]. Tradución de Luis G. Pérez Bueno. Astrágalo, núm. 7. Textos en Línea. En: http://textosenlinea.blogspot.com/2008/05/michel-foucault-losespacios-otros.html [Consulta: 20/10/2010].

FREUD, Sigmund (1999): El malestar en la cultura y otros ensayos. Traducción de Ramón Rey Ardid y Luis López Ballesteros. Madrid: Alianza.

GELFANT, Blanche H. (1974): "John Dos Passos: The Synoptic Novel". Hook, pp. 36-52.

GENETTE, Gérard (1989): Palimpsestos. La Literatura en Segundo Grado. Traducción de Celia Fernández Pietro. Madrid: Taurus.

HOOK, Andrew (ed.) (1974): Dos Passos. A Collection of Critical Essays. Englewood Cliffs, N. J.: Prentice-Hall, Inc. 
KAZIN, Alfred (1995): On Native Grounds. An Interpretation of Modern American Prose Literature. San Diego, New York, London: Harcourt Brace \& Company.

LEHAN, Richard (1998): The City in Literature. An Intellectual and Cultural History. Berkeley and Los Angeles: University of California Press.

LEUCHTENBURG, William E. (1958): The Perils of Prosperity, 1914-1932. Chicago and London: University of Chicago Press.

LOWRY, D. E. (1974): "Manhattan Transfer. Dos Passos' Wasteland". Hook, pp. 53-60.

MACLUHAN, Herbert Marshall (1974): "John Dos Passos: Tecnique vs. Sensibility". Hook, pp. 148-161.

MALORY, Sir Thomas (1969): Le Morte D'Arthur, vol. I, II. London: Penguin Books.

MOTTRAM, Eric (1971): "The Hostile Environment and the Survival Artist: A Note on the Twenties", en Malcolm Bradbury y D. Palmer (eds.), The American Novel in the Nineteen Twenties. London: Edward Arnold.

PIKE, Burton (1981): The Image of the City in Modern Literature. Princeton: Princeton University Press.

SCOTT, Jamie S.; y SIMPSON-HOUSLEY, Paul (1994): "Eden, Babylon, New Jerusalem: a Taxonomy for Writing the City", en Peter Preston y Paul Simpson-Housley (eds.), Writing the City: Eden, Babylon and the New Jerusalem, pp. 331-341. New York, London: Routledge.

SEE, Sam (2008): "Fast Book Read Slow: The Shape of Speed in Manhattan Transfer and The Sun Also Rises". Journal of Narrative Theory, núm. 38, vol. 3, pp. 342-377. En: http://muse.jhu.edu/journals/ jnt/summary/v038/38.3.see.html [Consulta: 13/10/2010].

SPENGLER, Oswald (1966): La Decadencia de Occidente, vol. 2. Traducción de Manuel G. Morente. Madrid: Espasa-Calpe.

VANDERWERKEN, David L. (1977): "Manhattan Transfer: Dos Passos' Babel Story". American Literature, núm. 49, vol. 2, pp. 253-267. En: JSTOR (http://www.jstor.org/stable/2925428). [Consulta: 10/10/2010]. 